\title{
RELAÇÃO ENTRE O ÍNDICE ESCLEROMÉTRICO E A RESISTÊNCIA À COMPRESSÃO DE CONCRETOS
}

\author{
NATALLI, JULIANA \\ Doutoranda em Engenharia Civil \\ Universidade Federal de Ouro Preto \\ Minas Gerais; Brasil \\ juliananatalli24gmail.com
}

MOREIRA, JOÃO VITOR

Graduando em Engenharia Civil

Universidade Federal de Ouro Preto

Minas Gerais; Brasil

jaomoreira-@hotmail.com

\section{FIGUEIREDO, ALINE}

Mestranda em Engenharia Civil

Universidade Federal de Ouro Preto

Minas Gerais; Brasil

alinesantanafigueiredo@gmail.com

\section{PEIXOTO, RICARDO}

Professor DSC. em Engenharia Civil

Universidade Federal de Ouro Preto

Minas Gerais; Brasil

fiorotti.ricardo@gmail.com

\author{
ANDRADE, HUMBERTO \\ Doutorando em Engenharia Civil \\ Universidade Federal de Ouro Preto \\ Minas Gerais; Brasil \\ andrade.hdias@gmail.com \\ COSTA, LAÍS \\ Doutoranda em Engenharia Civil \\ Universidade Federal de Ouro Preto \\ Minas Gerais; Brasil \\ lais.cristina.costa@gmail.com

\section{BATISTA, JÚNIO} \\ Técnico em edificações \\ Universidade Federal de Ouro Preto \\ Minas Gerais; Brasil \\ juniobatista07@gmail.com
}

\section{RESUMO}

A avaliação da resistência à compressão de elementos de concreto armado é necessária para alguns procedimentos construtivos, tais como aplicações de cargas construtivas, perícia e previsão da vida útil das estruturas. Métodos não destrutivos para a avaliação do concreto in loco tornam-se cada vez mais aplicados no mercado. Essas técnicas facilitam e otimizam as inspeções dos elementos de concreto, evitam danos à estrutura, reduzem custos e permitem aumentar significativamente o número de amostras. O esclerômetro é um equipamento que consiste em uma massa controlada por uma mola capaz de deslizar em torno de um pistão dentro de um alojamento tubular. $\mathrm{O}$ valor do rebote, em porcentagem da deformação inicial da mola, é denominada índice esclerométrico, utilizado para determinar a resistência à compressão. Esse trabalho tem como objetivo determinar curvas de correlação do esclerômetro obtidas por meio da correlação entre os índices esclerométricos e os resultados dos testes de compressão de corpos de prova cilíndricos para concretos de classes de resistência C30 e C35. Para cada projeto de mistura, foram produzidos 16 corpos de prova cilíndricos de dimensões $10 \times 20 \mathrm{~cm}$ (diâmetro x altura) para ensaios de resistência à compressão e um bloco de concreto armado de dimensões $50 \times 50 \times 50 \mathrm{~cm}$ (comprimento x altura x espessura) para testes de esclerometria. A partir do tratamento de dados fornecidos pelos ensaios, as curvas de correlação foram plotadas correlacionando as classes de resistência dos concretos, os índices esclerométricos e os resultados médios de resistência à compressão dos corpos de prova cilíndricos Palavras-chave: concreto, resistência à compressão, ensaios não destrutivos, esclerometria.

\begin{abstract}
The evaluation of the compressive strength of reinforced concrete elements is necessary for some construction procedures, such as constructive load applications, expertise and prediction of the structural life of the structures. Non-destructive methods for on-site concrete evaluation are becoming increasingly applied in the market. These techniques facilitate and optimize inspections of concrete elements, prevent damage to the structure, reduce costs and significantly increase the number of samples. A sclerometer is equipment consisting of a spring-controlled mass capable of sliding around a piston within a tubular housing. The rebound value, in percentage of the initial spring deformation, is called the sclerometric index, used to determine the compressive strength. This work aims to determine sclerometer calibration curves obtained through the correlation between the sclerometric indices and the results of the compression tests of cylindrical specimens
\end{abstract}


for concrete of strength classes C30 and C35. For each mixing project, 16 of dimensions $10 \times 20$ (diameter x height) cylindrical specimens were produced for compressive strength tests and one 50 x 50 x 50 (length x height $\mathrm{x}$ thickness) reinforced concrete block for sclerometry tests. From the data treatment provided by the tests, the calibration curves was plotted correlating the concrete strength classes, the sclerometric indices and the average compressive strength results of the cylindrical specimens.

Keywords: concrete, compressive strength, non-destructive tests, sclerometry.

\section{INTRODUÇÃO}

O concreto é o material construtivo mais utilizado no mundo devido a sua viabilidade técnica e econômica (Ali, et al., 2018; Güçlüer, 2020). A larga aplicação do material pode ser justificada pela sua capacidade de resistir a ação da água sem deterioração, diferentemente da madeira e do aço. Além disso, os elementos estruturais podem ser moldados em diversas formas e tamanhos, com baixo custo de transporte e produção, devido a disponibilidade de matérias-primas (Mehta \& Monteiro, 2014).

O controle da qualidade de estruturas de concreto armado é de grande importância para a avaliação de suas propriedades mecânicas, durabilidade e homogeneidade do material (Câmara, 2006). Esse controle tecnológico, cada vez mais exigido pelo mercado, pode ser realizado por meio de ensaios não destrutivos in loco, os quais não causam danos ao elemento ensaiado (Khan, 2012; Singh \& Singh, 2018). Ao contrário dos ensaios não destrutivos, os testes destrutivos são técnicas caras, em alguns casos de difícil acesso, e são representativas de um pequeno volume de concreto e de algum efeito localmente destrutivo na estrutura (Alwash, et al., 2016).

A aplicação de técnicas não destrutivas permitem detectar possíveis problemas e patologias que possam comprometer a estrutura, bem como avaliar a resistência à compressão em diversas idades (Singh \& Singh, 2018). Em projetos de estruturas de concreto armado, a resistência à compressão do concreto é especificada por seu valor característico aos 28 dias (Feng \& Li, 2015; Feng, et al., 2020). O valor desse parâmetro mecânico é verificado estatisticamente por ensaios de corpos de prova cilíndricos moldados durante a etapa de concretagem do elemento (DeRousseau, et al., 2019). No entanto, esse valor de resistência pode sofrer alterações que ocorrem devido as diferentes condições de lançamento, compactação e procedimentos de cura da matriz cimentícia. Devido a esse fato, pode surgir a necessidade de se estimar a resistência à compressão do concreto in loco na ocasião da desforma das peças (Câmara, 2006).

A avaliação da resistência à compressão de elementos de concreto armado torna-se necessária para verificação da capacidade estrutural de uma edificação quando, por exemplo, ocorre mudança no uso de uma estrutura (Alwash , et al., 2016). Há também necessidade de verificação desse parâmetro após uma falha estrutural, como no caso de danos causados por incêndio ou degradação ambiental (ACI 228.1R-03, 2003).

A esclerometria é um método não detrutivo que fornece um resultado rápido e preciso da resistência à compressão do concreto in loco (ACI 228.1R-03, 2003; Xu \& Li, 2018). O esclerômetro é um equipamento que consiste em uma massa controlada por uma mola capaz de deslizar em um pistão dentro de um alojamento tubular. O valor do rebote do equipamento na superfície do concreto ensaiado é utilizado para determinar a resistência à compressão (Xu \& Li, 2018).

No Brasil, o ensaio esclerométrico segue os procedimentos da NBR 7584 (ABNT, 2012), a qual prescreve uma área entre $8000 \mathrm{~mm}^{2}$ (aproximadamente $90 \mathrm{~mm}$ x $90 \mathrm{~mm}$ ) e $40000 \mathrm{~mm}^{2}(200 \mathrm{~mm}$ x $200 \mathrm{~mm}$ ). A região do elemento estrutural em que serão feitos os testes não destrutivos será preparada por meio de polimento enérgico e mapeada por pacometria para a identificação das barras de aço e cobrimento da armadura. A normativa também recomenda que a superfície a ser analisada esteja seca e isenta de poeira e pó superficial.

Em cada área de ensaio, devem ser efetuados dezesseis impactos, não sendo permitido mais de um impacto no mesmo ponto. A norma também recomenda que a distância mínima entre os centros de dois pontos seja de $30 \mathrm{~mm}$. Após a obtenção dos resultados do índice esclerométrico das dezesseis posições, calcula-se o índice esclerométrico médio, e a partir desse valor se obtém o índice esclerométrico efetivo. A resistência da peça estrutural pode ser estimada por meio de ábacos fornecidos pelo aparelho que relaciona o índice esclerométrico efetivo com a resistência à compressão do concreto.

No entanto, a utilização dessas curvas não é recomendada, uma vez que o esclerômetro pode não estar calibrado para a realização de ensaios em determinado tipo de concreto. O procedimento de execução mais correto é a obtenção de uma 
curva de correlação do concreto a ser inspecionado, a qual correlacine os resultados dos ensaios não destrutivos e a resistência à compressão do material (Breysse, 2012; Alwash , et al., 2016; Alwash, et al., 2017).

Dessa forma, esse estudo pretende determinar curvas de correlação do esclerômetro obtidas por meio da correlação entre os índices esclerométricos efetivos determinados em blocos de concreto, e os resultados dos testes de compressão de corpos de prova cilíndricos.

\section{MATERIAIS E MÉTODOS}

\subsection{Materiais}

Neste trabalho foram produzidos 2 tipos de concreto convencional: um classe C30 e outro classe C35. Para a execução do concreto C30 foi utilizado cimento Portland de alto-forno CP III-40RS, e para a produção do concreto C35 foi usado o cimento CP V-ARI de alta resistência inicial. O agregado miúdo utilizado nas matrizes convencionais foi areia natural de rio e o agregado graúdo foi a brita gnáissica com dimensão caracterítica de $25 \mathrm{~mm}$ (brita 1). As características físicas dos agregados são especificadas na Tabela 1 .

Tabela 1 - Características físicas dos agregados.

\begin{tabular}{|c|c|c|}
\hline Propriedade & Areia & Brita 1 \\
\hline Massa específica $\left(\mathrm{g} / \mathrm{cm}^{3}\right)$ & 2,65 & 2,64 \\
\hline Massa unitária $\left(\mathrm{g} / \mathrm{cm}^{3}\right)$ & 1,37 & 1,47 \\
\hline Módulo de finura & 2,36 & 7,0 \\
\hline Dimensão máxima característica $(\mathrm{mm})$ & 2,36 & 25,0 \\
\hline
\end{tabular}

Para a execução dos blocos de concreto foram fabricadas formas cúbicas com as dimensões de 50 x 50 x $50 \mathrm{~cm}$ (comprimento $\mathrm{x}$ altura $\mathrm{x}$ espessura) com chapas de compensado. Os blocos foram armados transversalmente com barras de diâmetro de 12,5 mm e longitudinalmente com barras do mesmo diâmetro. Foram utilizados espaçadores de $3 \mathrm{~cm}$ entre as formas e as barras para garantir os cobrimentos das peças produzidas.

\subsection{Projeto das misturas de concreto}

A dosagem dos concretos foi realizada utilizando software computacional (Soares, 2009) de acordo com o método ABCP considerando as classe C30 (30 MPa aos 28 dias) e C35 (35 MPa aos 28 dias). A consistência foi determinada pelo abatimento de tronco de cone fixado em $70 \pm 10 \mathrm{~mm}$. Na Tabela 2 são apresentados os traços (Cimento: Agregado miúdo: Agregado graúdo) de cada tipo de concreto.

Tabela 2 - Traço dos concretos executados.

\begin{tabular}{|c|c|c|c|}
\hline Concreto & Traço & Plastificante* & Fator a/c \\
\hline C30 & $1: 1,95: 3,05$ & $0,5 \%$ & 0,5 \\
\hline C35 & $1: 1,13: 2,01$ & $0,5 \%$ & 0,42 \\
\hline
\end{tabular}

\footnotetext{
* A quantidade de aditivo foi calculado em relação à massa de cimento Portland.
}

Para cada classe de resistência foram produzidas misturas com quantidade suficiente para a produção 16 corpos de prova (CP's) cilíndricos de dimensões 10 x 20 (diâmetro x altura) e um bloco de concreto com volume de $0,125 \mathrm{~m}^{3}$. Os CP's foram moldados em duas camadas adensadas com 12 golpes NBR5738 (ABNT, 2015) e os blocos foram moldados em três camadas adensadas com vibrador de imersão. Os CP's, desmoldados em 24 horas, e os blocos, desmoldados em 3 dias, foram curados ao ar livre. A Figura 1 mostra a concretagem dos blocos de concreto e o aspecto da peça após o desmolde. 

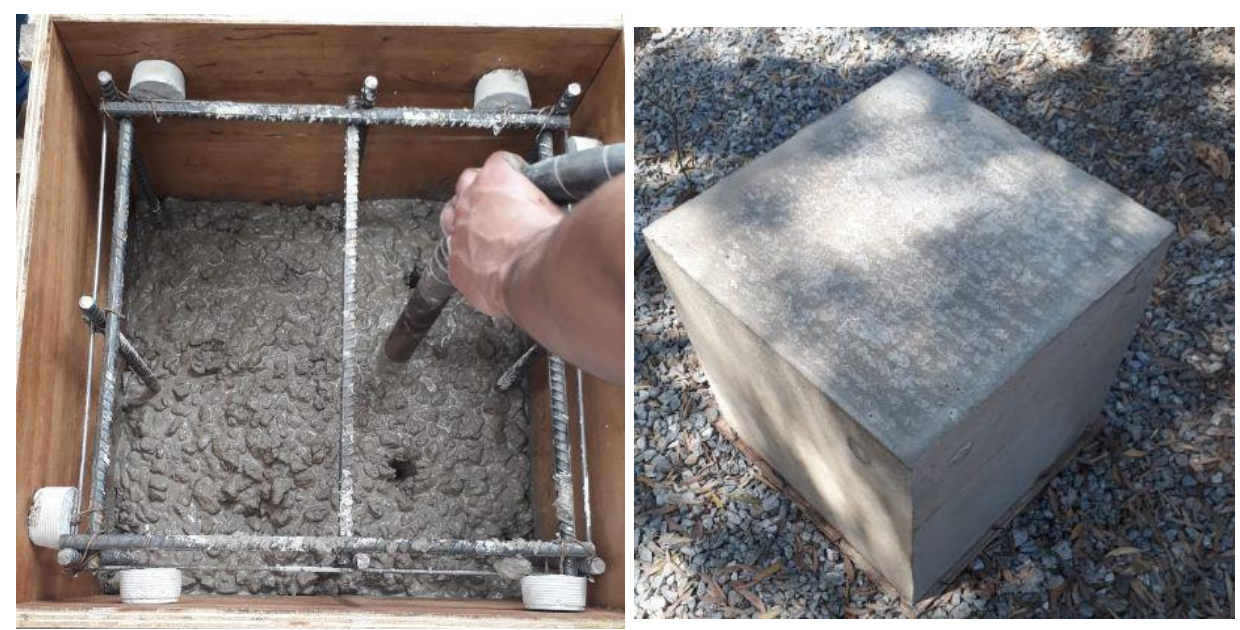

Figura 1 - Moldagem do bloco (à esquerda) e aspecto da estrutura após a desforma (à direita).

\subsection{Resistência à compressão dos corpos de prova}

Os ensaios de resistência dos CP's foram realizados em uma prensa servo-controlada modelo DL 20000-EMIC e PC 200 com carga máxima de $2000 \mathrm{kN}$ e velocidade de aplicação de carga de $0,45 \mathrm{MPa} / \mathrm{s}$. Para cada idade de 7, 21 e 28 dias quatro CP's foram ensaiados.

\subsection{Pacometria e esclerometria dos blocos de concreto}

As lateriais dos blocos de concreto, após serem preparadas por polimento enérgico e limpas, foram mapeadas por pacometria em equipamento Profoscope Bewehrungs-Sucher para a identificação da posição das barras de aço e cobrimento das armaduras.

Após a inspeção por pacometria, testes de esclerometria foram executados de acordo com procedimentos descritos na NBR 7584 (ABNT, 2012) por meio de esclerômetro modelo SilverSchmidt Type N, marca Proceq. Os ensaios não destrutivos foram realizados nas três laterais dos blocos cúbicos, sendo que cada lateral foi ensaiada para uma das idades de 7, 21 e 28 dias em 16 pontos de impactos. Após a obtenção dos resultados dos índices esclerométricos dos 16 pontos, foi desprezado todo índice esclerométrico individual que esteve afastado em mais de $10 \%$ do valor médio obtido, sendo posteriormente calculada uma nova média aritmética denominada $\left(\mathrm{I}_{\mathrm{m}}\right)$. A partir desse valor foi calculado o índice esclerométrico efetivo $\left(\mathrm{I}_{\mathrm{e}}\right.$ ), obtido pelo produto entre $\mathrm{I}_{\mathrm{m}} \mathrm{e} \mathrm{k}$ (constante do aparelho equivalente a 0,98 ). $\mathrm{O}$ coeficiente $\mathrm{k}$ foi calculado por meio de procedimentos descritos na NBR 7584 (ABNT, 2012).

\section{RESULTADOS E DISCUSSÕES}

\subsection{Resistência à compressão dos corpos de prova}

Os resultados obtidos para a resistência à compressão dos concretos são apresentados na Figura 2. Os dados à compressão dos concretos mostram que o concreto convencional C30 obteve aos 7 dias uma resistência equivalente a 24,93 MPa, apresentando um acréscimo de $30 \%$ aos 28 dias, alcançando um $\mathrm{f}_{\mathrm{cj}}$ esperado de $36,6 \mathrm{MPa}$ nessa idade. Como conhecido, esse acréscimo de resistência com a idade está relacionado a progressão da hidratação topoquímica dos grão de cimento Portland e a consequente formação de hidratos, especialmente do C-S-H (Taylor, 1997).

Assim como o C30, o concreto classe C35 atingiu o fcj esperado equivalente a 41,6 MPa aos 28 dias de idade. Pode-se observar que, aos 7 dias, a matriz alcançou uma resistência correspondente a 98,65\% da alcançada aos 28 dias. Isso ocorre, pois, os grãos de cimento Portland tipo CP-V ARI apresentam uma superfície específica bastante superior aos demais tipos de cimento, cerca de $300 \mathrm{~m}^{2} / \mathrm{kg}$ estabelecido por norma NBR 5733 (ABNT, 1991). Dessa forma, a velocidade do processo de hidratação da matriz é consideravelmente maior, atingindo altas resistências já nos primeiros dias de idade. 


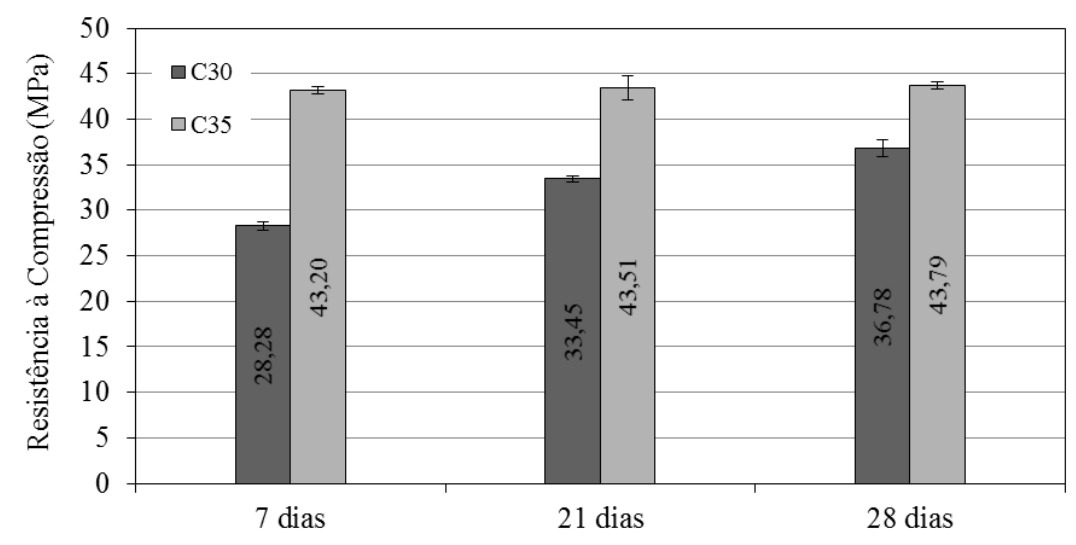

Figura 2 - Resistência à compressão dos corpos de prova.

\subsection{Esclerometria}

Os valores dos índices esclerométricos médios e efetivos dos blocos para as idades de 7, 21 e 28 dias são apresentados na Tabela 3 .

Tabela 3 - Índices esclerométricos dos blocos C30 e C35.

\begin{tabular}{|c|c|c|c|}
\hline Esclerometria & Idade (dias) & Im & Ie \\
\hline \multirow{3}{*}{ C30 } & 7 & 32,66 & 32,01 \\
\cline { 2 - 4 } & 21 & 34,19 & 33,51 \\
\cline { 2 - 4 } & 28 & 34,69 & 34,00 \\
\hline \multirow{3}{*}{$\mathbf{C 3 5}$} & 7 & 35,81 & 35,09 \\
\cline { 2 - 4 } & 21 & 39,88 & 39,08 \\
\cline { 2 - 4 } & 28 & 39,88 & 39,08 \\
\hline
\end{tabular}

Observa-se que, assim como os resultados de resistência à compressão, houve um crescente aumento dos índices esclerométricos efetivos ao longo das idades, atribuído ao aumento da rigidez das superfícies de concreto (Câmara, 2006; $\mathrm{Xu} \& \mathrm{Li}, 2018)$. Verifica-se também que os índices do bloco C35, para todas as idades, foram superiores ao do bloco C30, devido a maior taxa de hidratação do cimento Portland CP-V ARI e a consequente rigidez da peça obtida já nas primeiras idades. Pode-se observar também que, para as idades de 21 e 28 dias, os índices esclerométricos obtidos do bloco C35 foram idênticos, corroborando com os valores muito próximos de resistência à compressão dos CP's obtidos nessas mesmas idades.

\subsection{Curvas de correlação}

A partir dos resultados de resistência à compressão dos corpos de prova, e da esclerometria nas superfícies dos blocos foram plotadas as curvas de correlação do esclerômetro (Figura 3) para as duas classes de resistência especificadas de concreto convencional. A obtenção dessas curvas aumenta a eficiência da estimativa da resistência à compressão obtidas por meio de ensaios não destrutivos, melhorando, portanto, a confiabilidade dos resultados. 


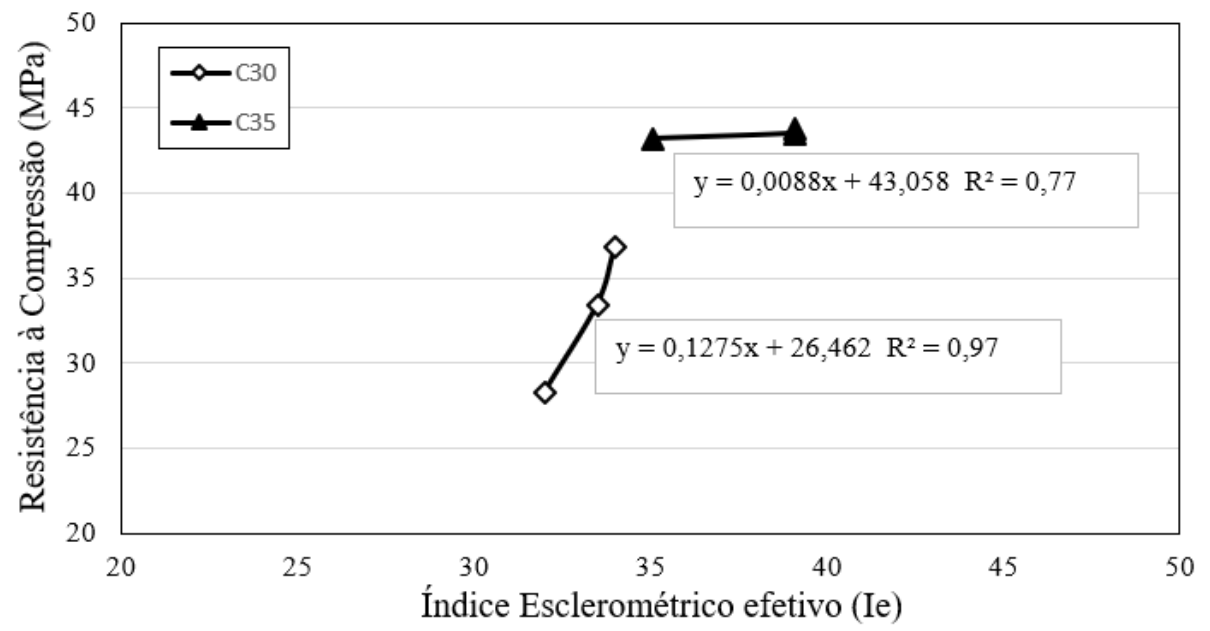

Figura 3 - Curvas de correlação dos concretos convencionais C30 e C35.

\section{CONCLUSÃO}

Esse artigo pretendeu criar curvas de correlação as quais relacionassem o índice esclerométrico efetivo $\left(\mathrm{I}_{\mathrm{e}}\right)$ com a resistência à compressão de corpos de prova 10 x $20 \mathrm{~cm}$ (diâmetro x altura) de concretos convencionais. Diante dos resultados apresentados, conclui-se que, para reduzir as incertezas dos resultados obtidos nos ensaios de esclerometria de concretos classes $\mathrm{C} 30$ e C35, produzidos respectivamente com CP-III e CP-V, pode-se fazer uso das curvas de correlação obtidas. Dessa forma, os valores fornecidos pela técnica não destrutiva se ajustarão de melhor forma aos dados experimentais.

\section{AGRADECIMENTOS}

O presente trabalho foi realizado com apoio da Coordenação de Aperfeiçoamento de Pessoal de Nível Superior - Brasil (CAPES) - Código de Financiamento 001. Os autores também agradecem as instituições FAPEMIG, CAPES, Fundação Gorceix, UFOP e CNPq pelo apoio para a realização e apresentação dessa pesquisa. Também somos gratos pela infraestrutura e colaboração do Grupo de Pesquisa em Resíduos Sólidos - RECICLOS - CNPq.

\section{REFERÊNCIAS}

ABNT, 1991. NBR 5733: Cimento Portland de alta resistência inicial, RJ: s.n.

ABNT, 2012. NBR 7584: Concreto endurecido - Avaliação da dureza superficial pelo esclerômetro de reflexão, RJ: ABNT.

ABNT, 2015. NBR 5738: Concreto - Procedimento para moldagem e cura de corpos de prova, RJ: ABNT. ACI 228.1R-03, 2003. In-place methods to estimate concrete strengths, s.1.: s.n.

Ali, M. R., Maslehuddin, M., Shameem, M. \& Barry, M. S., 2018. Thermal-resistant lightweight concrete with polyethylene beads as coarse aggregates. Constructions and Building Materials, Volume 164, pp. 739-749.

Alwash , M., Sbartaï, Z. M. \& Breysse, 2016. Non-destructive assessment of both mean strength and variability of concrete: A new bi-objective approach. Construction and Building Materials, Volume 113, pp. 880-889.

Alwash, M. et al., 2017. Factors affecting the reliability of assessing the concrete strength by rebound hammer and cores. Construction and Building Materials, Volume 140, pp. 354-363.

Breysse, D., 2012. Nondestructive evaluation of concrete strength: an historical review and new perspective by combining NDT methods. Construction and Building Materials, Volume 33, pp. 139-163. 
Câmara, E., 2006. Avaliação da resistência à compressão de concretos utilizados na grande Florianópolis através de ensaios não destrutivos, Santa Catarina: UFSC.

DeRousseau, M. A. et al., 2019. A comparison of machine learning methods for predicting the compressive strength of field-placed concrete. Construction and Building Materials, Volume 228, p. 116661.

Feng, D.-C. \& Li, J., 2015. Stochastic nonlinear behavior of reinforced concrete frames. ii: numerical simulation. Journal of Estructural Engineering, Volume 142.

Feng, D.-C.et al., 2020. Machine learning-based compressive strength prediction for concrete: An adaptive boosting approach. Construction and Building Materials, Volume 230, p. 117000.

Güçlüer, K., 2020. Investigation of the effects of aggregate textural properties on compressive strength (CS) and ultrasonic pulse velocity (UPV) of concrete. Journal of Building Engineering, Volume 27.

Khan, M. I., 2012. Evaluation of non-destructive testing of high strength concrete incorporating supplementary cementitious composites. Resources, Conservation and Recycling, Volume 61, pp. 125-129.

Mehta, P. \& Monteiro, P. J. M., 2014. Concrete: Microstructure, Properties and Materials. 2nd. ed. São Paulo: IBRACON.

Singh, N. \& Singh, S. P., 2018. Evaluating the performance of self compacting concretes made with recycled coarse and fine aggregates using non destructive testing techniques. Construction and Building Materials, Volume 181, pp. 73-84.

Soares, A. F. F., 2009. Software especialista para dosagem de misturas cimentícias, Belo Horizonte: Centro Federal de Educação Tecnológica de Minas Gerais.

Taylor, H., 1997. Cement Chemistry. 2nd. ed. London: Thomas Telford.

$\mathrm{Xu}, \mathrm{T} . \& \mathrm{Li}, \mathrm{J} ., 2018$. Assessing the spatial variability of the concrete by the rebound hammer test and compression test of drilled cores. Construction and Building Materials, Volume 188, pp. 820-832. 